\title{
Polyunsaturated fatty acids, lung function, and health- related quality of life in patients with chronic obstructive pulmonary disease
}

\author{
Hyunji Choi ${ }^{1}$, Taeyun Kim ${ }^{2}$ \\ ${ }^{1}$ Department of Laboratory Medicine, Kosin University Gospel Hospital, Busan, Korea \\ ${ }^{2}$ Division of Pulmonology, Department of Internal Medicine, Kosin University Gospel Hospital, Busan, Korea
}

Received: February 17, 2020

Revised: March 26, 2020

Accepted: March 26, 2020

Corresponding author:

Taeyun Kim

Division of Pulmonology,

Department of Internal Medicine,

Kosin University Gospel Hospital,

262 Gamcheon-ro, Seo-gu, Busan

49267, Korea

Tel: $+82-51-990-5820$

Fax: +82-51-990-5820

E-mail:jimsb89@naver.com
Background: Dietary polyunsaturated fatty acids (PUFA) are thought to modify systemic inflammation. The present study aimed to evaluate the relationship between PUFA intake, lung function, and health-related quality of life (HROoL) in patients with chronic obstructive pulmonary disease (COPD).

Methods: In this study, we used the dataset of 6th Korea National Health and Nutrition Examination Survey, in which, a total of 22,948 individuals including 573 participants with a high probability of developing COPD were enrolled. Participants with missing data for the investigated variables were excluded. Linear regression analyses were used to evaluate the association between PUFA intake (omega-3 [N3], omega-6 [N6], and total) with lung function, and HROoL. HROoL was determined according to the European Quality of Life-5 Dimensions (EQ-5D). Subgroup analysis of older patients was performed. Age, sex, body mass index, smoking, alcohol, education, residence, total calorie intake, and predicted $\mathrm{FEV}_{1} \%$ were adjusted in all analyses.

Results: Although lung function was not associated with PUFA intake, EQ-5D index was remarkably associated with $\mathrm{N} 3, \mathrm{N6}$, and total PUFA intake in a dose-dependent manner. This association was more pronounced in elderly COPD patients. Mean levels of N3, N6, and total PUFA intake were significantly higher in patients having better HROoL with respect to mobility, self-care, and usual activities.

Conclusion: Our results suggest that N3, N6, and total PUFA intake are associated with $\mathrm{HROoL}$ in COPD patients. This association may be attributed to mobility, self-care, and usual activities. Further longitudinal study is required to clarify this relationship.

Keywords: Chronic obstructive pulmonary disease; Korea National Health and Nutrition Examination Survey; Polyunsaturated fatty acid; Quality of life

\section{Introduction}

Chronic obstructive pulmonary disease (COPD) is a preventable and treatable inflammatory disease characterized by persistent respiratory symptoms and airflow limitation measured by spirome- try [1]. The disease burden of COPD is steadily increasing, not only in western countries but also in Asian countries [2]. Since COPD requires constant management, it imposes substantial social, economic, and medical burdens. For example, a recent multicenter observational study in Korea showed that 1,245.6 million

Copyright (C) 2020 Yeungnam University College of Medicine

This is an Open Access article distributed under the terms of the Creative Commons Attribution Non-Commercial License (http://creativecommons.org/licenses/by-nc/4.0/) which permits unrestricted non-commercial use, distribution, and reproduction in any medium, provided the original work is properly cited. 
US dollars were required to provide COPD-related direct and indirect medical services [3].

The pathogenesis of COPD involves airway and systemic inflammatory response [4]. Individuals exposed to noxious particles may develop airway inflammation with loss of terminal and transitional bronchioles, emphysematous destruction, and lung function declines [5]. Systemic inflammation is associated with poor clinical outcome. For instance, Agusti et al. [6] evaluated systemic inflammatory biomarkers in peripheral blood and showed that the increased inflammatory reaction in COPD patients is associated with increased all-cause mortality and exacerbation frequency. Patients with severe disease have an elevated inflammatory burden, as they usually experience more rapid decline in lung function, increase in severity of symptoms, and frequent exacerbations [7]. Therefore, it is important to alleviate inflammatory response not only in the airway, but also in the circulatory system.

Polyunsaturated fatty acids (PUFA) play a role in modifying inflammation [8]. For example, a study involving 80 patients with COPD, who received $9 \mathrm{~g}$ of PUFA or placebo for 8 weeks, demonstrated improvement in exercise capacity [9]. Although recent data from a study on US adults with COPD showed that omega-3 (N3) PUFA was associated with respiratory symptoms [10], this association was not investigated among Korean COPD patients.

In this context, the present study aimed to evaluate the association between dietary PUFA, including total, N3, omega-6 (N6) PUFA, and disease severity as well as the HRQoL in patients with COPD using data from a nationwide representative sample survey.

\section{Material and methods}

\section{Ethics statatement}

The survey protocol of the Korea National Health and Nutrition Examination Survey (KNHANES) was approved by the Institutional Review Board of the KCDC (IRB No: 2013-07CON-03$4 C$ in 2013, 2013-12EXP-03-5C in 2014). Since the KNHANES of 2015 was conducted for public welfare, approval of the IRB was not required. Written informed consent was obtained from all participants before the survey, which was conducted according to the Declaration of Helsinki. All procedures were in accordance with the Strengthening the Reporting of Observational Studies in Epidemiology (STROBE) statement for observational studies.

\section{Study participants}

The present study used the dataset of the 6th (2013-2015) KNHANES. The KNHANES is an annually conducted nationwide population-based cross-sectional survey by the Korea Centers for
Disease Control and Prevention (KCDC) and the Korean Ministry of Health and Welfare. The KNHANES was designed as a complex sample survey using a multistage sampling method to represent the general non-institutionalized Korean population. The dataset of the KNHANES is freely accessible online, and detailed survey profiles are described in a previous report [11].

The 2013-2015 KNHANES assessed the health and nutritional status of 29,321 South Koreans, and 22,948 responded to the survey (response rate 78.3\%). The KNHANES collected health-related and nutritional information by evaluating laboratory samples, physical examinations, face-to-face interviews, and nutritional consumption. Pulmonary function testing (PFT) was performed in participants aged over 40 years; thus, 10,109 participants without PFT results were excluded. Participants with missing values in other variables (i.e., residential area, education level, smoking status, alcohol consumption, body mass index [BMI], and European Quality of Life-5 Dimensions [EQ-5D], and nutrition intake) were excluded $(n=6,408)$. Participants diagnosed as asthma by their physician or having medication for asthma were excluded $(n=112)$. Participants with the value of forced expiratory volume in 1 second $\left(\mathrm{FEV}_{1}\right)$ divided by forced vital capacity (FVC) above $70 \%$ were excluded, due to the low probability of having COPD $(n=5,746)$. Finally, 573 participants with a high probability of having COPD without missing values in the possible confounding variables were included in the analysis. The study flow chart is presented in Fig. 1.

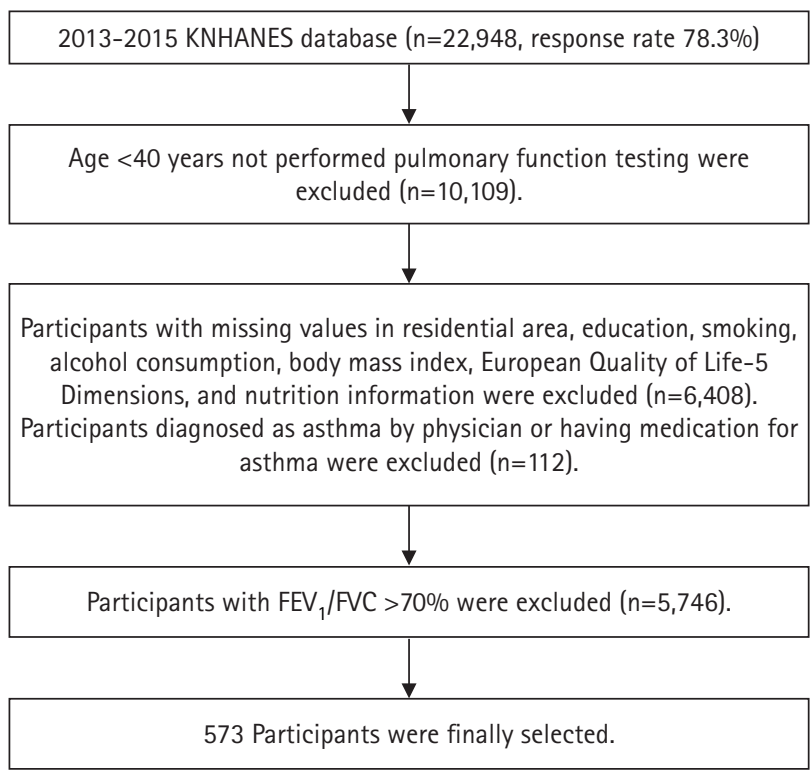

Fig. 1. Flow chart of the study using the dataset of the 6th Korea National Health and Nutrition Examination Survey (KNHANES). $\mathrm{FEV}_{1}$, forced expiratory volume in 1 second; FVC, forced vital capacity. 


\section{Data collection and measurements}

PFT was performed using dry rolling seal spirometers (Model 2130; SensorMedics, Yorba Linda, CA, USA) by highly-trained medical technicians. The quality control of the PFT was conducted according to the standardization guidelines of the American Thoracic Society and the European Respiratory Society [12]. Participants with $\mathrm{FEV}_{1} / \mathrm{FVC}<70 \%$ were considered to have COPD based on the classification of the Global Initiative for Chronic Obstructive Lung Disease (GOLD) 2020 report. Indices of lung function in participants with COPD included $\mathrm{FEV}_{1}(\mathrm{~L})$, predicted $\mathrm{FEV}_{1} \%$, $\mathrm{FVC}(\mathrm{L})$, predicted $\mathrm{FVC} \%, \mathrm{FEV}_{1} / \mathrm{FVC} \%$, and peak expiratory flow (PEF, $\mathrm{L} / \mathrm{sec})$.

Smoking status was categorized into three groups (current, former, and never) based on the Centers for Disease Control and Prevention classification [13]. Current smokers were defined as participants who smoked more than 100 cigarettes in his/her lifetime and smoke currently. Former smokers were defined as participants who smoked more than 100 cigarettes in his/her lifetime; however, had stopped smoking for more than 1 year. Participants who never smoked were defined as participants who never smoked or smoked less than 100 cigarettes in his/her lifetime.

The EQ-5D was used to evaluate the HRQoL in participants with COPD. The validity and usefulness of the EQ-5D for measuring the quality of life in COPD patients have been demonstrated previously [14]. The EQ-5D consists of five dimensions to measure the quality of life: mobility, self-care, usual activity, pain/ discomfort, and anxiety/depression. The participants were asked to select one of the three following responses for each of the five dimensions: G1, no problem; G2, some problems; G3, severe problems. Furthermore, the EQ-5D index was used to evaluate the dose-dependent relationship between PUFA intake and HRQoL. The formula for the EQ-5D index has been described in a previous report by Nam et al. [15], with higher scores indicating higher HRQoL.

Dietary PUFA intake was measured using a 24-hour recall method. The 24-hour recall method is a structured interview for estimating the intake of food or drink that an individual participant consumed during the day. Although the method can have a day-to-day variability, the reliability, validity, and reproducibility have been previously demonstrated [16]. The amounts of dietary N3 PUFA (g/day), N6 PUFA (g/day), and total PUFA (g/day) were measured.

Data regarding socio-economic status and anthropometric indices were measured by trained survey assistants. Residential areas were categorized into two groups: urban and rural. Educational level was categorized into three groups: middle school or less, high school, and college or more. Heavy alcohol consumption was defined as $\geq 7$ drinks in men and $\geq 5$ drinks in women on an occasion. The participants were categorized into two groups: whether they consumed more than one heavy alcohol drink in a week, or not. BMI was categorized based on the Korean Society for the Study of Obesity guidelines [17]: underweight $(<18.5$ $\left.\mathrm{kg} / \mathrm{m}^{2}\right)$, normal $\left(18.5-22.9 \mathrm{~kg} / \mathrm{m}^{2}\right)$, pre-obese $\left(23-24.9 \mathrm{~kg} / \mathrm{m}^{2}\right)$, and obese $\left(\geq 25 \mathrm{~kg} / \mathrm{m}^{2}\right)$.

\section{Statistical analysis}

Since the KNHANES was conducted using a complex, multistage, stratified, and probability verified sample design, all statistical analyses were performed under complex sample analyses, and sampling weights were applied. The sample, therefore, represents the non-institutionalized South Korean population.

Continuous variables (age and spirometric values) were compared using the ANOVA test and presented as a mean value with a standardized error. Categorical variables (residence, education, smoking status, alcohol consumption, and BMI) were compared using a chi-square test and presented as percentages with standard errors. Post-hoc analysis was performed with Bonferroni correction, and $p<0.017$ was considered statistically significant between groups.

HRQoL was categorized into five dimensions according to the EQ-5D as mobility, self-care, usual activities, pain/discomfort, and anxiety/depression. The mean estimated level and standard error of PUFA intake in the G1, G2, and G3 groups were calculated using linear regression analysis. The trend for significance between PUFA intake and HRQoL was evaluated using linear regression analysis considering the G1, G2, and G3 of HRQoL as a continuous variable.

Multivariable linear regression analyses were used to evaluate the dose-dependent relationship between N3, N6, total PUFA intake, and spirometric values $\left(\mathrm{FEV}_{1} \%\right.$ predicted, $\mathrm{FVC} \%$ predicted, and PEF). The association between PUFA intake and HRQoL (EQ-5D index) was measured using linear regression analysis. Model 1 was adjusted for age and sex. Model 2 was adjusted for age, sex, BMI, smoking status, and alcohol consumption. Model 3 was adjusted for age, sex, BMI, smoking status, alcohol consumption, residence, education, total calorie intake, and predicted $\mathrm{FEV}_{1} \%$. Prior to placing these variables into the model, a multi-collinearity test was performed in order to identify any inter-correlations among the investigated variables. As the distribution of N3, N6, and total PUFA intake violated the assumption of normality and skewed left, the value was log-transformed. Subgroup analysis was performed in elderly COPD patients.

All statistical analyses were performed with IBM SPSS version 24.0 (IBM Corp., Armonk, NY, USA). For all analyses, a $p$-val- 
ue $<0.05$ was considered statistically significant.

\section{Results}

Baseline characteristics of study participants are presented (Table 1). The relationships between N3, N6, and total PUFA intake and lung function are presented (Table 2). Lung function, which was measured using predicted $\mathrm{FEV}_{1} \%$, FVC\%, and $\mathrm{PEF}$, was not significantly associated with PUFA intake. The association between PUFA intake and HRQoL is presented (Table 3 ). The estimated amounts of N3, N6, and total PUFA intake were significantly associated with mobility and self-care ability in a dose-dependent manner, and statistical significance remained after covariate ad-

Table 1. Baseline characteristics of study participants $(n=573)$

\begin{tabular}{|c|c|}
\hline Characteristic & Total \\
\hline Age (yr) & $57.7 \pm 0.5$ \\
\hline Man $(\%)$ & $63.6 \pm 2.2$ \\
\hline \multicolumn{2}{|l|}{ Residential area (\%) } \\
\hline Urban & $78.3 \pm 2.4$ \\
\hline Rural & $21.7 \pm 2.4$ \\
\hline \multicolumn{2}{|l|}{ Education } \\
\hline Middle school or less & $40.8 \pm 2.3$ \\
\hline High school & $37.7 \pm 2.2$ \\
\hline College or more & $21.6 \pm 1.9$ \\
\hline \multicolumn{2}{|l|}{ Smoking status } \\
\hline Current & $31.1 \pm 2.3$ \\
\hline Former & $30.7 \pm 2.3$ \\
\hline Never & $38.1 \pm 2.3$ \\
\hline \multicolumn{2}{|c|}{ Heavy alcohol consumption (/wk) } \\
\hline$<1$ & $64.8 \pm 2.2$ \\
\hline$\geq 1$ & $35.2 \pm 2.2$ \\
\hline \multicolumn{2}{|c|}{ Body mass index $\left(\mathrm{kg} / \mathrm{m}^{2}, \%\right)$} \\
\hline$<18.5$ & $2.2 \pm 0.7$ \\
\hline $18.5-22.9$ & $39.5 \pm 2.4$ \\
\hline $23-24.9$ & $27.0 \pm 2.2$ \\
\hline$\geq 25.0$ & $31.2 \pm 2.2$ \\
\hline \multicolumn{2}{|l|}{ Spirometric value } \\
\hline $\mathrm{FEV}_{1}(\mathrm{~L})$ & $2.3 \pm 0.0$ \\
\hline $\mathrm{FEV}_{1} \%$, predicted & $75.5 \pm 0.7$ \\
\hline $\mathrm{FVC}(\mathrm{L})$ & $3.7 \pm 0.0$ \\
\hline FVC\%, predicted & $92.1 \pm 0.7$ \\
\hline $\mathrm{FEV}_{1} / \mathrm{FVC} \%$ & $62.9 \pm 0.4$ \\
\hline PEF (L) & $6.0 \pm 0.1$ \\
\hline
\end{tabular}

Values are presented as mean \pm standard error (SE) or weighted percentage \pm SE.

$\mathrm{FEV}_{1}$, forced expiratory volume in 1 second; $\mathrm{FVC}$, forced vital capacity; PEF, peak expiratory flow.

a) Heavy alcohol consumption was defined as $\geq 7$ drinks in men and $\geq 5$ drinks in women on an occasion. justment. N3, N6, and total PUFA intake also showed a positive association with the capacity of usual activities, although the degree of significance varied with covariates adjustment. Although N3 PUFA intake was positively associated with pain/discomfort, N6 and total PUFA intake were not. With respect to anxiety/depression, statistical significance was not observed. The associations between N3, N6, and PUFA intake and the EQ-5D index are presented (Table 4). High PUFA intake was significantly associated with a better EQ-5D score after covariate adjustment. Despite statistical significance, the magnitude of association was low. The association in older COPD patients is presented (Table 5). The strength of association was intensified in older patients, and the same statistical significance was observed.

\section{Discussion}

In a population-based sample from South Korea, we investigated the association between PUFA intake, lung function, and HRQoL in patients with COPD defined as $\mathrm{FEV}_{1} / \mathrm{FVC} \%<70 \%$. We adjusted for socio-economic status, health behaviors, total calorie intake, and predicted $\mathrm{FEV}_{1} \%$, and found that $\mathrm{N} 3, \mathrm{~N} 6$, and total PUFA intake were associated with HRQoL in a dose-dependent manner. Specifically, high dodes of N3, N6, and total PUFA intake showed a positive association with mobility, self-care, and usual activities in COPD patients, whereas there was no significant association with pain/discomfort and anxiety/depression. In a subgroup analysis of older patients (age $\geq 60$ years), this association was reinforced, supporting the importance of proper nutritional supplements in older populations. Our results provide insight regarding the association of nutrition intake and HRQoL in patients with COPD.

HRQoL has been an important primary outcome in several studies and focus areas in the management of COPD. Triple inhaled treatments with long-acting beta-agonists, long-acting anticholinergics, and inhaled corticosteroids demonstrated improvement of HRQoL in COPD patients [18]. The evaluation of treatment efficacy and detection of an individual patient's potential risk for psychological and behavioral problems should be feasible by assessing their HRQoL [19]. The EQ-5D is a useful descriptive methodology evaluating five dimensions of HRQoL: mobility, self-care, usual activities, pain/discomfort, and anxiety/depression. In a previous study measuring the usefulness of the EQ-5D score in COPD patients, the correlation of hospital admissions, comorbidities, COPD Assessment Test scores, and the Medical Research Council scale for dyspnea was observed [20]. Pickard et al. [21] compared nine structured research criteria with the validity and reliability of EQ-5D usage in COPD patients and reported 
Table 2. Association between lung function parameters and PUFA intake $(n=573)$

\begin{tabular}{|c|c|c|c|c|c|c|}
\hline \multirow{2}{*}{ Variable } & \multicolumn{2}{|c|}{ Predicted $\mathrm{FEV}_{1} \%$} & \multicolumn{2}{|c|}{ Predicted FVC\% } & \multicolumn{2}{|c|}{ PEF (L/sec) } \\
\hline & Coefficient \pm SE & $p$-value & Coefficient \pm SE & $p$-value & Coefficient \pm SE & $p$-value \\
\hline \multicolumn{7}{|l|}{ Total PUFA } \\
\hline Model 1 & $0.228 \pm 1.874$ & 0.903 & $-1.152 \pm 1.860$ & 0.536 & $0.177 \pm 0.182$ & 0.332 \\
\hline Model 2 & $0.394 \pm 1.897$ & 0.835 & $-0.806 \pm 1.880$ & 0.668 & $0.195 \pm 0.189$ & 0.303 \\
\hline Model 3 & $-0.458 \pm 1.889$ & 0.809 & $-1.748 \pm 1.844$ & 0.344 & $0.083 \pm 0.188$ & 0.661 \\
\hline \multicolumn{7}{|l|}{ N3 PUFA } \\
\hline Model 1 & $0.970 \pm 1.647$ & 0.556 & $0.203 \pm 1.659$ & 0.903 & $0.196 \pm 0.156$ & 0.207 \\
\hline Model 2 & $1.022 \pm 1.701$ & 0.549 & $0.440 \pm 1.681$ & 0.794 & $0.209 \pm 0.162$ & 0.197 \\
\hline Model 3 & $0.545 \pm 1.745$ & 0.894 & $-0.303 \pm 1.650$ & 0.854 & $0.173 \pm 0.162$ & 0.286 \\
\hline \multicolumn{7}{|l|}{ N6 PUFA } \\
\hline Model 1 & $-0.145 \pm 1.804$ & 0.936 & $-1.411 \pm 1.764$ & 0.424 & $0.145 \pm 0.177$ & 0.413 \\
\hline Model 2 & $0.022 \pm 1.821$ & 0.99 & $-1.085 \pm 1.781$ & 0.543 & $0.161 \pm 0.182$ & 0.376 \\
\hline Model 3 & $-0.845 \pm 1.813$ & 0.642 & $-1.946 \pm 1.752$ & 0.267 & $0.033 \pm 0.181$ & 0.854 \\
\hline
\end{tabular}

Intake amount of total, N3, and N6 PUFA was log-transformed, because of left-skewed distribution. Model 1 was adjusted for age and sex; model 2 was additionally adjusted for body mass index, smoking status, and alcohol consumption; and model 3 was additionally adjusted for residence, education, and total calorie intake.

PUFA, polyunsaturated fatty acid; FEV 1 , forced expiratory volume in 1 second; $F V$, forced vital capacity; PEF, peak expiratory flow; SE, standard error; N3, omega-3; N6, omega-6.

Table 3. Estimated amount (g) of daily PUFA intake in COPD patients according to degree of health-related problems (G1, G2, and G3)

\begin{tabular}{|c|c|c|c|c|c|c|c|c|c|c|c|c|}
\hline \multirow{2}{*}{ Variable } & \multicolumn{4}{|c|}{ N3 PUFA } & \multicolumn{4}{|c|}{ N6 PUFA } & \multicolumn{4}{|c|}{ Total PUFA } \\
\hline & G1 & G2 & G3 & $p$-value & G1 & G2 & G3 & $p$-value & G1 & G2 & G3 & $p$-value \\
\hline $\begin{array}{l}\text { Mobility } \\
\text { (prevalence, \%) }\end{array}$ & $82.6 \pm 1.8$ & $6.9 \pm 1.8$ & $0.6 \pm 0.3$ & & $32.6 \pm 1.8$ & $16.9 \pm 1.8$ & $0.6 \pm 0.3$ & & $82.6 \pm 1.8$ & $16.9 \pm 1.8$ & $0.6 \pm 0.3$ & \\
\hline Model $1^{\text {b) }}$ & $73 \pm 0.1$ & $16 \pm 0.1$ & $1.15 \pm 0.7$ & $<0.001$ & $68 \pm 0.4$ & $6.53 \pm 0.6$ & $4.01 \pm 1.4$ & $<0.001$ & $10.32 \pm 0.4$ & $7.64 \pm 0.6$ & $5.19 \pm 2.0$ & $<0.001$ \\
\hline Model 2 & $64 \pm 0.1$ & $.08 \pm 0.1$ & $1.03 \pm 0.7$ & $<0.001$ & $22 \pm 0.4$ & $6.23 \pm 0.6$ & $3.45 \pm 1.4$ & $<0.001$ & $9.79 \pm 0.5$ & $7.27 \pm 0.6$ & $4.52 \pm 1.9$ & $<0.001$ \\
\hline Mode & $4 \pm 0.1$ & $.06 \pm 0.1$ & $.99 \pm 0.7$ & & $50 \pm 0.5$ & $6.73 \pm 0.6$ & $3.55 \pm 1.8$ & & $10.09 \pm 0.6$ & $7.77 \pm 0.8$ & $4.60 \pm 2.3$ & 0.001 \\
\hline $\begin{array}{l}\text { Self-care } \\
\text { (prevalence, } \% \text { ) }\end{array}$ & $5.0 \pm 0.9$ & $5.0 \pm 0.9$ & - & & $95.0 \pm 0.9$ & $5.0 \pm 0.9$ & - & & $95.0 \pm 0.9$ & $5.0 \pm 0.9$ & - & \\
\hline & $65 \pm 0.1$ & $14 \pm 0.2$ & - & 0.006 & $38 \pm 0.3$ & $6.28 \pm 0.8$ & - & & $9.95 \pm 0.4$ & $7.33 \pm 0.9$ & - & 0.004 \\
\hline Mode & $6 \pm 0.1$ & $08 \pm 0.2$ & - & & $92 \pm 0.4$ & $6.04 \pm 0.8$ & - & & $9.42 \pm 0.4$ & $7.04 \pm 0.9$ & - & 0.011 \\
\hline & $5 \pm 0.1$ & $5 \pm 0.2$ & - & & $3 \pm 0.5$ & $.48 \pm 0.8$ & - & & $.73 \pm 0.5$ & $7.47 \pm 0.9$ & - & 0.011 \\
\hline $\begin{array}{l}\text { Usual act } \\
\text { (prevaler }\end{array}$ & $9.4 \pm 1.5$ & $10.2 \pm 1.5$ & $0.4 \pm 0.3$ & & $89.4 \pm 1.5$ & $10.2 \pm 1.5$ & $0.4 \pm 0.3$ & & $89.4 \pm 1.5$ & $10.2 \pm 1.5$ & $0.4 \pm 0.3$ & \\
\hline & $68 \pm 0.1$ & $19 \pm 0.1$ & $1.12 \pm 0.3$ & $<0.001$ & $.45 \pm 0.4$ & $6.85 \pm 0.8$ & $5.40 \pm 1.0$ & & $10.05 \pm 0.4$ & $7.95 \pm 0.9$ & $6.44 \pm 1.2$ & 0.01 \\
\hline & $8 \pm 0.1$ & $10 \pm 0.1$ & $1.03 \pm 0.3$ & & $99 \pm 0.4$ & $6.52 \pm 0.8$ & $5.32 \pm 0.9$ & & $.52 \pm 0.4$ & $7.54 \pm 0.9$ & $6.28 \pm 1.1$ & 0.01 \\
\hline Model 3 & $58 \pm 0.1$ & $1.09 \pm 0.1$ & $1.06 \pm 0.3$ & 0.001 & $29 \pm 0.5$ & $6.98 \pm 0.8$ & $5.96 \pm 0.7$ & 0.075 & $9.82 \pm 0.5$ & $8.00 \pm 0.9$ & $6.95 \pm 0.9$ & 0.027 \\
\hline $\begin{array}{l}\text { Pain/discomfort } \\
\text { (prevalence, \%) }\end{array}$ & $74.7 \pm 2.0$ & $22.7 \pm 1.9$ & $2.6 \pm 0.6$ & & $74.7 \pm 2.0$ & $22.7 \pm 1.9$ & $2.6 \pm 0.6$ & & $74.7 \pm 2.0$ & $22.7 \pm 1.9$ & $2.6 \pm 0.6$ & \\
\hline Model 1 & $1.70 \pm 0.1$ & $48 \pm 0.1$ & $1.00 \pm 0.2$ & 0.026 & $3.37 \pm 0.4$ & $8.05 \pm 0.8$ & $7.36 \pm 1.4$ & & $10.00 \pm 0.4$ & $9.42 \pm 0.9$ & $8.27 \pm 1.5$ & 0.358 \\
\hline & $1.60 \pm 0.1$ & $39 \pm 0.1$ & $0.96 \pm 0.2$ & & $88 \pm 0.5$ & $7.66 \pm 0.8$ & $7.26 \pm 1.4$ & & $44 \pm 0.5$ & $8.95 \pm 0.8$ & $8.14 \pm 1.5$ & 0.476 \\
\hline Model 3 & $1.59 \pm 0.1$ & $1.36 \pm 0.1$ & $0.99 \pm 0.2$ & 0.046 & $.16 \pm 0.5$ & $8.02 \pm 0.8$ & $8.10 \pm 1.4$ & 0.893 & $9.71 \pm 0.6$ & $9.31 \pm 0.9$ & $9.01 \pm 1.5$ & 0.614 \\
\hline $\begin{array}{l}\text { Anxiety/de- } \\
\text { pression } \\
\text { (prevalence, \%) }\end{array}$ & $85.7 \pm 1.8$ & $13.1 \pm 1.7$ & $1.3 \pm 0.5$ & & $85.7 \pm 1.8$ & $13.1 \pm 1.7$ & $1.3 \pm 0.5$ & & $85.7 \pm 1.8$ & $13.1 \pm 1.7$ & $1.3 \pm 0.5$ & \\
\hline Model 1 & $1.59 \pm 0.1$ & $1.94 \pm 0.3$ & $0.37 \pm 0.1$ & 0.805 & $8.23 \pm 0.4$ & $8.89 \pm 1.0$ & $4.17 \pm 0.7$ & & $9.74 \pm 0.4$ & $10.83 \pm 1.2$ & $4.55 \pm 0.8$ & 0.988 \\
\hline & $1.51 \pm 0.1$ & $1.87 \pm 0.3$ & $0.36 \pm 0.1$ & & $7.79 \pm 0.4$ & $8.53 \pm 1.0$ & $4.38 \pm 0.7$ & & $9.23 \pm 0.5$ & $10.40 \pm 1.2$ & $4.72 \pm 0.8$ & 0.882 \\
\hline Model 3 & $1.49 \pm 0.1$ & $1.87 \pm 0.3$ & $0.37 \pm 0.2$ & 0.705 & $8.05 \pm 0.5$ & $8.96 \pm 1.1$ & $5.42 \pm 0.9$ & 0.768 & $9.48 \pm 0.6$ & $10.85 \pm 1.2$ & $5.79 \pm 1.0$ & 0.687 \\
\hline
\end{tabular}

Comparison of estimated PUFA intake and $p$-value for trend was measured using multivariable linear regression analysis considering G1, G2, and G3 as continuous variables. Model 1 was adjusted for age and sex; model 2 was additionally adjusted for body mass index, smoking status, and alcohol consumption; and model 3 was additionally adjusted for residence, education, total calorie intake, and predicted $\mathrm{FEV}_{1} \%$.

PUFA, polyunsaturated fatty acid; COPD, chronic obstructive pulmonary disease; $\mathrm{G} 1$, no problem; $\mathrm{G} 2$, some problems; G3, severe problems; N3, omega-3; N6, omega-6; FEV , forced expiratory volume in 1 second.

Estimated prevalence of subjects and amount of PUFA intake according to degree of health-related problems are presented as ${ }^{\text {a) }}$ percentage \pm standard error and ${ }^{b)}$ estimated amount of intake \pm standard error, respectively. 
Table 4. Association of PUFA intake with HRQoL in COPD patients $(n=573)$

\begin{tabular}{|c|c|c|c|c|c|c|}
\hline \multirow{2}{*}{ Variable } & \multicolumn{2}{|c|}{ Total PUFA } & \multicolumn{2}{|c|}{ N3 PUFA } & \multicolumn{2}{|c|}{ N6 PUFA } \\
\hline & Coefficient \pm SE & $p$-value & Coefficient \pm SE & $p$-value & Coefficient \pm SE & $p$-value \\
\hline Crude & $0.877 \pm 0.147$ & $<0.001$ & $0.813 \pm 0.168$ & $<0.001$ & $0.914 \pm 0.150$ & $<0.001$ \\
\hline Model 1 & $0.586 \pm 0.141$ & $<0.001$ & $0.621 \pm 0.171$ & $<0.001$ & $0.602 \pm 0.142$ & $<0.001$ \\
\hline Model 2 & $0.549 \pm 0.141$ & $<0.001$ & $0.596 \pm 0.171$ & 0.001 & $0.562 \pm 0.144$ & $<0.001$ \\
\hline Model 3 & $0.409 \pm 0.132$ & 0.002 & $0.472 \pm 0.153$ & 0.002 & $0.417 \pm 0.139$ & 0.003 \\
\hline
\end{tabular}

Multivariable linear regression analyses were performed after log-transforming the value of total, N3, and N6 PUFA because of its left-skewed distribution. HROoL was measured using European Quality of Life-5 Dimensions. Model 1 was adjusted for age and sex; model 2 was additionally adjusted for body mass index, smoking status, and alcohol consumption; and model 3 was additionally adjusted for residence, education, total calorie intake, and predicted $\mathrm{FEV}_{1} \%$.

PUFA, polyunsaturated fatty acid; HROoL, health-related quality of life; COPD, chronic obstructive pulmonary disease; N3, omega-3; N6, omega-6; SE, standard error; FEV $_{1}$, forced expiratory volume in 1 second.

Table 5. Association of PUFA intake on HRQoL in elderly COPD patients $(n=298)$

\begin{tabular}{|c|c|c|c|c|c|c|}
\hline \multirow{2}{*}{ Variable } & \multicolumn{2}{|c|}{ Total PUFA } & \multicolumn{2}{|c|}{ N3 PUFA } & \multicolumn{2}{|c|}{ N6 PUFA } \\
\hline & Coefficient \pm SE & $p$-value & Coefficient \pm SE & $p$-value & Coefficient \pm SE & $p$-value \\
\hline Crude & $0.838 \pm 0.186$ & $<0.001$ & $0.797 \pm 0.221$ & $<0.001$ & $0.865 \pm 0.186$ & $<0.001$ \\
\hline Model 1 & $0.627 \pm 0.197$ & 0.002 & $0.528 \pm 0.224$ & 0.019 & $0.673 \pm 0.201$ & 0.001 \\
\hline Model 2 & $0.592 \pm 0.193$ & 0.002 & $0.481 \pm 0.215$ & 0.026 & $0.642 \pm 0.198$ & 0.001 \\
\hline Model 3 & $0.532 \pm 0.186$ & 0.005 & $0.354 \pm 0.175$ & 0.045 & $0.532 \pm 0.186$ & 0.005 \\
\hline
\end{tabular}

Multivariable linear regression analyses were performed after log-transforming the value of total, N3, and N6 PUFA because of its left-skewed distribution.HROoL was measured using European Quality of Life-5 Dimensions. Model 1 was adjusted for age and sex; model 2 was additionally adjusted for body mass index, smoking status, and alcohol consumption; and model 3 was additionally adjusted for residence, education, total calorie intake, and predicted $\mathrm{FEV}_{1} \%$.

PUFA, polyunsaturated fatty acid; HROoL, health-related quality of life; COPD, chronic obstructive pulmonary disease; N3, omega-3; N6, omega-6; SE, standard error; $\mathrm{FEV}_{1}$, forced expiratory volume in 1 second.

that the EQ-5D scores were closely related to COPD stages.

Among the five components of the EQ-5D, mobility is defined as the ability to walk, while usual activities refer to an individual's performance at work, study, household, and family/leisure activities; these two components are associated with daily physical activities. Walking ability in COPD patients is further standardized by the measurement of the distance covered by walking in $6 \mathrm{~min}$ utes, and lower mean walking distance is generally considered as a poor prognostic factor for mortality [22]. The GOLD report recommends exercise testing and assessment of physical activity based on walking distance to evaluate the effectiveness of pulmonary rehabilitation, which is a key non-pharmacologic management technique for COPD patients [23]. Given that physical activity is a strong predictor of all-cause mortality in COPD patients [22], our results suggest that PUFA intake may correlate with mobility and usual physical activities. However, specific threshold amounts of PUFA for improving physical activity is unclear and further longitudinal study is required.

Several previous studies have shown that PUFA is associated with HRQoL. In patients with systemic lupus erythematosus
(SLE) from the United States, N3 PUFA intake, calculated from a diet history questionnaire, was found to be beneficial in patient-reported outcomes assessed by the Systemic Lupus Activity Questionnaire [24]. One randomized controlled trial of $1 \mathrm{~g} /$ day N3 PUFA supplementation demonstrated a reduction of premenstrual symptoms and improvement of HRQoL [25]. Another double-blind randomized controlled trial of $3 \mathrm{~g} /$ day N3 PUFA supplementation for 3 months reported a reduction in several inflammatory markers and improvement of HRQoL in chronic hemodialysis patients [26]. Although the association between PUFA and HRQoL in patients with COPD is under-recognized, Lemoine $S$ et al. [10] suggested that individual factors should be considered while determining the association of N3 PUFA intake and symptoms. PUFA may be beneficial in other chronic diseases such as SLE or chronic kidney disease. PUFA may also have beneficial effects in patients with COPD in terms of HRQoL.

There are several possible mechanisms related to this association. First, COPD is a chronic airway and systemic inflammatory disease, and N3 PUFA might attenuate this inflammatory process. Within the inflammatory processes of the human body, N3 
PUFA, eicosapentaenoic acid and docosahexaenoic acid mediate anti-inflammatory responses and several specialized pro-resolving mediators (SPMs, resolvins, protectins, and maresins) are synthesized [8]. Resolvin, one of the SPMs, has been associated with counter-regulated pro-inflammatory signaling and inflammatory resolution pathways in patients with COPD [27]. In a cigarette exposed human lung, an experimental study demonstrated that resolvin dampened the inflammatory reaction via the production of anti-inflammatory cytokines and enhanced phagocytosis of macrophages [27]. Second, the susceptibility of PUFA to oxidative stress could contribute to lowering airway inflammation. Interestingly, we observed that N6 PUFA was beneficial in improving HRQoL in COPD patients, although there is conflicting evidence regarding the health-related benefits of N6 PUFA [28,29]. PUFA can be easily oxidized due to its unstable hydrogen-carbon bonds; an in vivo study demonstrated that N6 PUFA decreased serum C-reactive protein [29].

Our study has several strengths. This is the first population-based epidemiologic study in South Korea showing a relationship between PUFA intake and HRQoL in patients with COPD. These results highlight the importance of nutrient intake in patients with COPD to alleviate respiratory symptoms and improve HRQoL. In addition, we adjusted for socio-economic characteristics and health-related behavior, as diet is influenced by an individual's status, as well as social, economic, and cultural factors.

Despite these strengths, our study has several limitations. First, as this is a cross-sectional observational study, the causal relationship is unclear. Although we observed that PUFA intake is associated with increased HRQoL in COPD patients, the inverse correlation could exist. Second, because the nutritional survey of KNHANES was based on a 24-hour recall method, recall bias and day-to-day variability should be considered. Third, spirometric values were obtained through pre- bronchodilator tests. Fourth, information regarding respiratory symptoms, hospital admission or exacerbation history, infections within the 4 weeks prior to the study, and recent use of corticosteroid was unavailable. Therefore, classification based on symptoms or exacerbation (e.g., ABCD grouping of the GOLD report) was not feasible. Fifth, which PUFA derivative is specifically associated with HRQoL in unclear. For example, Noguchi et al. [30] reported that eicosapentaenoic acid might improve the quality of life; however, docosahexaenoic acid was not beneficial. Finally, smoking status in terms of packyears is an important component when assessing long-term inflammation and oxidative stress, but the data was not available in 6th KNHANES data.

N3, N6, and total PUFA showed a positive correlation with HRQoL in patients with COPD. Specifically, mobility, self-care, and usual activities might be attributable to the observed association between HRQoL and PUFA intake. Further randomized prospective studies are required to clarify the health-related benefits of PUFA in patients with COPD.

\section{Acknowledgments}

\section{Conflicts of interest}

No potential conflict of interest relevant to this article was reported.

\section{Author contributions}

Conceptualization: HC, TK; Data curation and Formal analysis: TK; Investigation, Methodology, Project administration, and Supervision: TK; Validation: HC, TK; Visualization: TK; Writing-original draft: HC; Writing-review \& editing: TK.

\section{ORCID}

Hyunji Choi, https://orcid.org/0000-0002-6453-7099

Taeyun Kim, https://orcid.org/0000-0001-7786-5051

\section{References}

1. Viegi G, Pistelli F, Sherrill DL, Maio S, Baldacci S, Carrozzi L. Definition, epidemiology and natural history of COPD. Eur Respir J 2007;30:993-1013.

2. Zhu B, Wang Y, Ming J, Chen W, Zhang L. Disease burden of COPD in China: a systematic review. Int J Chron Obstruct Pulmon Dis 2018;13:1353-64.

3. Kim C, Kim Y, Yang DW, Rhee CK, Kim SK, Hwang YI, et al. Direct and indirect costs of chronic obstructive pulmonary disease in Korea. Tuberc Respir Dis (Seoul) 2019;82:27-34.

4. Barnes PJ. Inflammatory mechanisms in patients with chronic obstructive pulmonary disease. J Allergy Clin Immunol 2016; 138:16-27.

5. MacNee W. Pathogenesis of chronic obstructive pulmonary disease. Proc Am Thorac Soc 2005;2:258-66.

6. Agusti A, Edwards LD, Rennard SI, MacNee W, Tal-Singer R, Miller BE, et al. Persistent systemic inflammation is associated with poor clinical outcomes in COPD: a novel phenotype. PLoS One 2012; 7:e37483.

7. Kim V, Aaron SD. What is a COPD exacerbation? Current definitions, pitfalls, challenges and opportunities for improvement. Eur Respir J 2018;52:1801261

8. Buckley CD, Gilroy DW, Serhan CN. Proresolving lipid mediators and mechanisms in the resolution of acute inflammation. Immunity 2014;40:315-27. 
9. Broekhuizen R, Wouters EF, Creutzberg EC, Weling-Scheepers CA, Schols AM. Polyunsaturated fatty acids improve exercise capacity in chronic obstructive pulmonary disease. Thorax 2005;60:376-82.

10. Lemoine S CM, Brigham EP, Woo H, Hanson CK, McCormack MC, Koch A, et al. Omega-3 fatty acid intake and prevalent respiratory symptoms among U.S. adults with COPD. BMC Pulm Med 2019;19:97.

11. Kweon S, Kim Y, Jang MJ, Kim Y, Kim K, Choi S, et al. Data resource profile: the Korea National Health and Nutrition Examination Survey (KNHANES). Int J Epidemiol 2014;43:69-77.

12. Miller MR, Hankinson J, Brusasco V, Burgos F, Casaburi R, Coates A, et al. Standardisation of spirometry. Eur Respir J 2005;26:319-38.

13. Centers for Disease Control and Prevention (CDC). Vital signs: nonsmokers' exposure to secondhand smoke. United States, 1999-2008. MMWR Morb Mortal Wkly Rep 2010;59:1141-6.

14. Nolan CM, Longworth L, Lord J, Canavan JL, Jones SE, Kon SS, et al. The EQ-5D-5L health status questionnaire in COPD: validity, responsiveness and minimum important difference. Thorax 2016;71:493-500.

15. Nam H, Kim K, Kwon S, Koh K, Poul K. EQ-5D Korean valuation study using time trade off method. Seoul (KR): Korea Centers for Disease Control and Prevention; 2007.

16. Karvetti RL, Knuts LR. Validity of the 24-hour dietary recall. J Am Diet Assoc 1985;85:1437-42.

17. Seo MH, Lee WY, Kim SS, Kang JH, Kang JH, Kim KK, et al. 2018 Korean Society for the Study of Obesity guideline for the management of obesity in Korea. J Obes Metab Syndr 2019; 28:40-5.

18. Singh D, Vestbo J. Triple therapy in chronic obstructive pulmonary disease. Am J Respir Crit Care Med 2017;196:1082-3.

19. Hawthorne G, Richardson J, Osborne R. The Assessment of Quality of Life (AQoL) instrument: a psychometric measure of health-related quality of life. Qual Life Res 1999;8:209-24.

20. Esquinas C, Ramon MA, Nunez A, Molina J, Quintano JA, Roman-Rodriguez $M$, et al. Correlation between disease severity factors and EQ-5D utilities in chronic obstructive pulmonary disease. Qual Life Res 2020;29:607-17.

21. Pickard AS, Willke C, Jung E, Patel S, Stavem K, Lee TA. Use of a preference-based measure of health (EQ-5D) in COPD and asthma. Respir Med 2008;102:519-36.

22. Cote CG, Pinto-Plata V, Kasprzyk K, Dordelly LJ, Celli BR. The 6-min walk distance, peak oxygen uptake, and mortality in COPD. Chest 2007;132:1778-85.

23. Singh D, Agusti A, Anzueto A, Barnes PJ, Bourbeau J, Celli BR, et al. Global strategy for the diagnosis, management, and prevention of chronic obstructive lung disease: the GOLD science committee report 2019. Eur Respir J 2019;53:1900164.

24. Charoenwoodhipong P, Harlow SD, Marder W, Hassett AL, McCune WJ, Gordon C, et al. Dietary omega polyunsaturated fatty acid intake and patient-reported outcomes in systemic lupus erythematosus: the Michigan Lupus Epidemiology and Surveillance Program. Arthritis Care Res (Hoboken). 2020;72:874-81.

25. Behboudi-Gandevani S, Hariri FZ, Moghaddam-Banaem L. The effect of omega 3 fatty acid supplementation on premenstrual syndrome and health-related quality of life: a randomized clinical trial. J Psychosom Obstet Gynaecol 2018;39:266-72.

26. Moeinzadeh F, Shahidi S, Mortazavi M, Dolatkhah S, Kajbaf M, Haghjooy Javanmard S, et al. Effects of omega-3 fatty acid supplementation on serum biomarkers, inflammatory agents, and quality of life of patients on hemodialysis. Iran J Kidney Dis 2016;10:381-7.

27. Croasdell A, Thatcher TH, Kottmann RM, Colas RA, Dalli J, Serhan CN, et al. Resolvins attenuate inflammation and promote resolution in cigarette smoke-exposed human macrophages. Am J Physiol Lung Cell Mol Physiol 2015;309:L888901.

28. Baylin A, Campos H. Arachidonic acid in adipose tissue is associated with nonfatal acute myocardial infarction in the central valley of Costa Rica. J Nutr 2004;134:3095-9.

29. Kaikkonen JE, Kresanov P, Ahotupa M, Jula A, Mikkila V, Viikari JS, et al. High serum n6 fatty acid proportion is associated with lowered LDL oxidation and inflammation: the Cardiovascular Risk in Young Finns Study. Free Radic Res 2014;48: 420-6.

30. Noguchi H, Nishi D, Matsumura K, Hamazaki K, Hamazaki T, Matsuoka YJ. Limited effect of omega-3 fatty acids on the quality of life in survivors of traumatic injury: a randomized, placebo-controlled trial. Prostaglandins Leukot Essent Fatty Acids 2017;127:1-5. 